\title{
Kontroversen bei CED
}

\begin{abstract}
Wer Patienten mit Colitis ulcerosa oder Morbus Crohn behandelt, weiß nur zu gut, dass es häufig nicht das eine richtige Therapieregime gibt. Ziel des Falk Seminars "CED kontrovers" ist es deshalb, Pros und Kontras zu strittigen Themen darzustellen und zu diskutieren. Eine der Fragestellungen: Soll die Therapiesteuerung nach subjektiven oder objektiven Kriterien erfolgen?
\end{abstract}

Symptomatik und objektive Befunde, wie etwa Entzündungsparameter oder Endoskopie passen bei chronisch entzündlichen Darmerkrankungen (CED) längst nicht immer zusammen. Manche Patienten klagen über starke Beschwerden ohne positiven Laborbefund. Andere fühlen sich wohl, trotz florider Kolitis. Und nun? „Wir behandeln nicht die Entzündungsmarker, sondern den Patienten“, machte Prof. Dr. Andreas Stallmach, Jena, seine Position deutlich und plädierte damit für eine Steuerung der Behandlung nach subjektiven Kriterien. Dabei verwies er auf die mangelnde Korrelation objektiver Befunde mit der Krankheits- aktivität. Auch Inflammationsmarker im Normbereich seien kein Garant für eine zu vernachlässigende Krankheitsaktivität: „Ein normaler CRP-Wert schließt eine Entzündung nicht aus.“ Bei Patienten mit Beschwerden ohne objektiven positiven Befund sollte allerdings abgeklärt werden, ob der Symptomatik möglicherweise eine andere Ursachen zugrunde liegt, wie etwa ein distales Kurzdarmsyndrom, eine bakterielle Fehlbesiedelung oder auch ein Reizdarm. PD Dr. Jürgen Büning, Lübeck, forderte bei dieser Konstellation eine ausführliche Diagnostik, auch um ein malignes Geschehen nicht zu übersehen. Liegt der Fall umgekehrt, hat der Patient also keine Symptome, aber erhöhte Entzündungsparameter und/ oder einen positiven endoskopischen $\mathrm{Be}$ fund, muss zwischen Übertherapie und Komplikationsrisiko abgewogen werden. Büning setzte sich für eine effektive Therapie auch bei beschwerdefreien Patienten ein. Sein Argument: Die mukosale Heilung geht mit weniger Schüben, weniger Krankenhausaufenthalten, weniger Komplikationen und weniger Operationen einher. Stallmach wies dagegen auf das erhöhte Infektionsrisiko als Folge einer Therapieintensivierung hin, welches in das Gesamtkalkül einbezogen werden muss.

\section{Stenose bei Morbus Crohn: Dilatation oder Operation?}

Soll bei einer Patientin, die eine Stenose im terminalen Ileum mit einer reizlosen Fistelöffnung entwickelt hat, endoskopisch dilatiert oder operiert werden? Für ein konservatives Vorgehen mit endoskopischer Dilatation plus Immunsuppression sprach sich Prof. Dr. Christian Maaser, Lüneburg, aus mit Verweis auf das hohe Rezidivrisiko nach einem operativen Eingriff. Innerhalb der darauffolgenden 15 Jahre muss bei mehr als der Hälfte der Patienten mit einem Rezidiv und einer zweiten Resektion gerechnet wer- den. Die endoskopische Dilatation zeige dagegen auch langfristig gute Ergebnisse. Bei einem medianen Follow-up von 5,8 Jahren konnte $76 \%$ der Patienten die Operation erspart werden - bei geringen Nebenwirkungen. Auch aktuell publizierte Daten stützen die endoskopische Dilatation. Bei $86 \%$ von 1.500 behandelten Patienten mit stenosierendem Morbus Crohn wurde Durchgängigkeit erreicht, eine klinische Besserung bei $80 \%$. Als günstige Ausgangssituation für die endoskopische Dilatation plus antient-

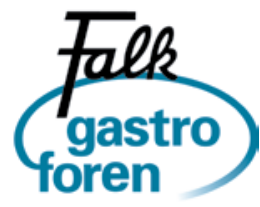

Besuchen Sie das nächste Falk Gastro Forum „Viszeralmedizin in Jena - Standards und aktuelle Entwicklungen“ am Samstag, den 28. November 2015 in Jena; Infos: www.drfalkpharma.de/veranstaltungen

zündliche Medikation nannte Maaser Stenosen mit einer Länge von maximal $4 \mathrm{~cm}$, eine echoarme Wandschichtung, kein Darmkonglomerat sowie kein Anhalt für Abszedierung. Prof. Dr. Ekkehard Jehle, Ravensburg, sieht in der Operation dagegen die Chance auf Heilung und er betonte „Frei sein von Chirurgie ist kein Therapieziel!" Lebensqualität und Symptomfreiheit seien die eigentlichen Therapieziele. Beides lasse sich mit einem chirurgischen Eingriff erreichen. Gegen die endoskopische Dilatation führte er verschiedene Punkte an: viele Stenosen sind endoskopisch nicht erreichbar, häufig liegen mehrere Stenosen vor, die prätherapeutisch nicht bekannt sind, oder die Stenosen sind vernarbt und damit nicht dilatierbar.

(BF)

Falk Seminar "CED kontrovers", Stuttgart, 4. Juli 2015 